\title{
Analysis of factors associated with unintended initial dissection of the posterior plane during small incision lenticule extraction
}

\author{
Ke Zheng ${ }^{1,2,3 \#}$, Tian Han ${ }^{1,2,3 \#}$, Yinan Han ${ }^{2}$, Fang Liu ${ }^{1,2,3}$, Xingtao Zhou ${ }^{1,2,3}$ \\ ${ }^{1}$ Eye Institute and Department of Ophthalmology, Eye \& ENT Hospital, Fudan University, Shanghai, China; ${ }^{2}$ NHC Key Laboratory of \\ Myopia (Fudan University), Key Laboratory of Myopia, Chinese Academy of Medical Sciences, Shanghai, China; ${ }^{3}$ Shanghai Research Center of \\ Ophthalmology and Optometry, Shanghai, China \\ Contributions: (I) Conception and design: All authors; (II) Administrative support: All authors; (III) Provision of study materials or patients: None; \\ (IV) Collection and assembly of data: All authors; (V) Data analysis and interpretation: All authors; (VI) Manuscript writing: All authors; (VII) Final \\ approval of manuscript: All authors. \\ \#These authors contributed equally to this work. \\ Correspondence to: Xingtao Zhou. 83\# Fenyang Road/19\# Baoqing Road, Shanghai 200031, China. Email: doctzhouxingtao@163.com.
}

Background: To investigate potential risk factors for unintended initial dissection of the posterior plane in the initial learning curve of small incision lenticule extraction (SMILE).

Methods: Data were derived from consecutive 263 eyes of 136 patients who underwent SMILE at the beginning of the surgeon's learning curve. Probabilities of unintended initial dissection of the posterior plane in left and right eyes were analyzed. Preoperative sphere, cylinder, spherical equivalent (SE), J0, J45, lenticular diameter, lenticular thickness, corneal thickness, and axial length were assessed between eyes in which posterior plane dissection was unintended and those in which it was not.

Results: Unintended initial dissection of the posterior plane occurred in 29 eyes, corresponding to an incidence rate of $11.03 \%$. The probability of difficulty in plane dissection in the left eye was significantly higher than that in the right eye $(\mathrm{P}=0.003)$. Significant differences were found in sphere $(\mathrm{T}=2.8, \mathrm{P}=0.006)$, SE $(\mathrm{T}=2.37, \mathrm{P}=0.019), \mathrm{J} 0(\mathrm{~T}=2.05, \mathrm{P}=0.043)$ and axial length $(\mathrm{T}=-2.79, \mathrm{P}=0.006)$ between eyes with and without unintended initial dissection of the posterior plane.

Conclusions: In the present study, difficulty in plane dissection during SMILE was encountered significantly more often in left eyes for a right-hand doctor, and in eyes with low SE or high J0 values. Special attention should be paid to prevent unintended initial dissection of the posterior plane in such situations.

Keywords: Femtosecond laser; lenticule dissection; small incision lenticule extraction (SMILE)

Submitted Oct 06, 2020. Accepted for publication Jan 17, 2021.

doi: $10.21037 / \mathrm{atm}-20-6759$

View this article at: http://dx.doi.org/10.21037/atm-20-6759

\section{Introduction}

In recent years, femtosecond laser technology has been widely applied in ophthalmology. Femtosecond laser small incision lenticule extraction (SMILE) has demonstrated good safety, effectiveness, stability, and predictability $(1,2)$. Compared with traditional refractive surgery, SMILE has two substantial advantages. One is that corneal flap is eliminated, effectively preventing flap-related complications; The other is that the comparatively smaller incision size leads to milder postoperative reactions $(3,4)$, less nerve damage $(5,6)$, and a reduced likelihood of dry eye $(7,8)$.

Notably, however, SMILE is also associated with some specific surgical risks and intraoperative complications due to such a small incision $(9,10)$. Surgeons with relatively little experience are prone to encounter lenticule dissection and extraction difficulties. Lenticule dissection and extraction in SMILE requires a high level of surgical skill. To shorten 
the learning curve of SMILE, consensuses on SMILE established by experts in China and other countries (11) suggest that lenticule dissection should be performed via the sequential dissection of anterior lenticular interface, and then posterior interface of the lenticule. Differentiating between the two interfaces represents the most challenging step of the entire surgical procedure. Novice surgeons are prone to initially dissect the posterior plane of the lenticule prior to dissection of the anterior plane. Such a situation is known as unintended dissection of the posterior plane. When it occurs, the lenticule dissection becomes difficult due to the transparent nature of the lenticule and the adhesion of the lenticule to the corneal cap $(12,13)$.

In the present study, potential risk factors for unintended initial dissection of the posterior plane in the initial learning curve of SMILE were analyzed. We present the following article in accordance with the TRENT reporting checklist (available at http://dx.doi.org/10.21037/atm-20-6759).

\section{Methods}

\section{Subjects}

Consecutive patients who underwent SMILE by the same surgeon at the beginning of his learning curve were included in this retrospective study. Inclusion criteria for surgery were age $\geq 18$ years, spherical equivalent (SE) $\leq-12.5 \mathrm{D}$, corrected distance visual acuity $\geq 20 / 20$, stable myopia for at least 2 years, and no contact lens wearing for at least 2 weeks. Exclusion criteria were the presence or history of eye conditions other than myopia and astigmatism, such as keratoconus or external eye injury, a history of eye surgery, and the presence or history of a systemic disease.

The study was conducted in accordance with the Declaration of Helsinki, and it was approved by the ethics committee of the Eye and ENT Hospital of Fudan University (KJ2010-18). Written informed consent was obtained from all the patients.

\section{Surgical procedure}

All standard SMILE procedures were performed by the same surgeon (KZ). The surgeon in the present study have finished more than 100 LASIK, LASEK and PRK. The $500-\mathrm{kHz}$ VisuMax femtosecond laser system (Carl Zeiss Meditec, Jena, Germany) was used with a pulse energy of $130 \mathrm{~nJ}$ and spot separation of $4.5 \mu \mathrm{m}$. The cap diameter was set to $7.5 \mathrm{~mm}$ at a 100 - to $120-\mu \mathrm{m}$ depth. A $90^{\circ}$ single side cut (12 o'clock) with a length of $2 \mathrm{~mm}$ (60 degrees) was created during the procedure. The minimum thickness of lenticules was 10 to $20 \mu \mathrm{m}$. The transition zone for the astigmatic treatments was $0.1 \mathrm{~mm}$.

During the dissection, the surgeon used the left hand to control the eye with forceps and used the right hand to dissect the anterior interface with a spatula (model No.52435T; 66 Vision-tech Corp., Suzhou, China) followed by the posterior interface through the superior incision directly, without pocketing the two interfaces of the lenticule edge first.

\section{Collection of patient information}

The information recorded included the occurrence of unintended dissection of the posterior plane, subjective refraction results (sphere, cylinder, SE, J0 and J45), axial length, corneal thickness, lenticular diameter, and lenticular thickness. Corneal thickness was measured using a Scheimpllug imaging system (Pentacam HR, Type 70900, Germany).

\section{Statistical analysis}

All data were analyzed using SAS 9.4 (SAS Institute Inc., Cary, NC, USA). The Pearson's chi-squared test was used to compare the probabilities of unintended dissection of the posterior plane in the left or right eyes. A linear mixed model was used to analyse factors associated with unintended dissection of the posterior plane. In all tests, differences were considered statistically significant when $\mathrm{P}<0.05$.

\section{Results}

Data were derived from 263 eyes of 136 patients. The mean age was $22.82 \pm 4.65$ years and the mean SE was $-5.67 \pm 2.03 \mathrm{D}$ (Table 1). During the surgery, no obvious suction loss, black spot or opaque bubble layer affected the operation.

Unintended dissection of the posterior plane occurred in 29 eyes, corresponding to an incidence rate of $11.03 \%$. Nine patients underwent monovision surgery, and unintended dissection of the posterior plane occurred in 1 of those 9 eyes, while in the other 127 patients, it occurred in 28 eyes. Eventually, all lenticules were separated from the anterior plane successfully and were extracted integrally.

The probability of unintended dissection of the posterior plane in the left eye was significantly higher than that in the 
Table 1 Demographic and preoperative clinical data $(n=263)$

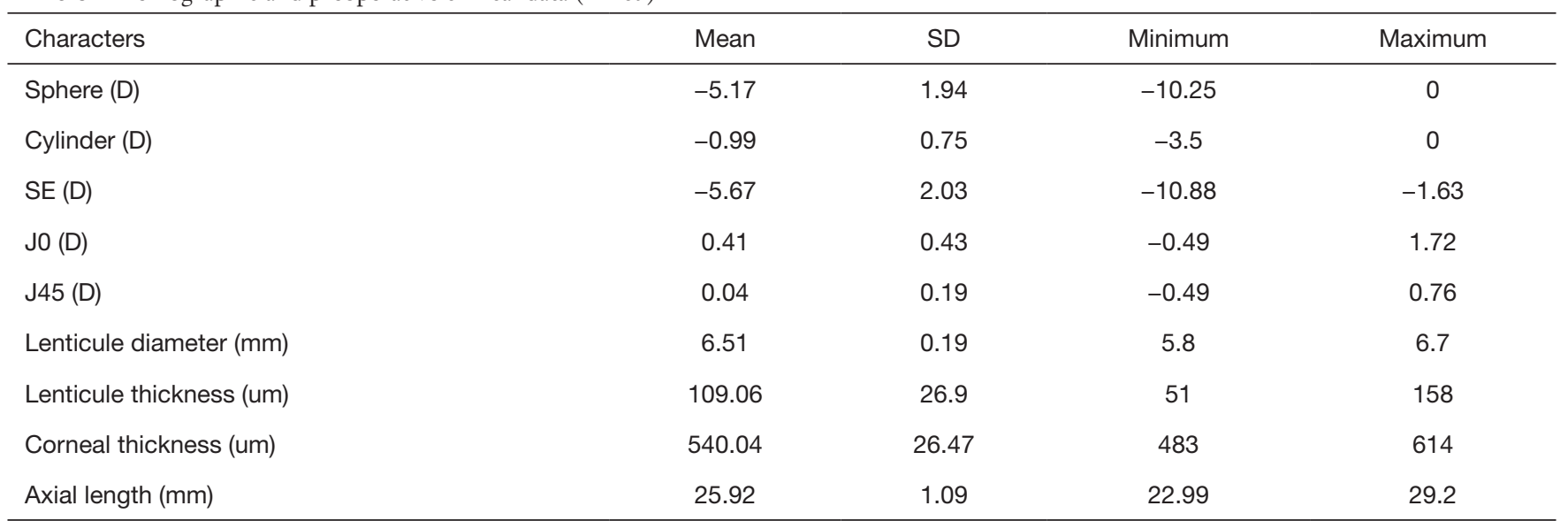

$\mathrm{D}$, diopters.

Table 2 Comparisons of right and left eyes with and without unintended initial dissection of the posterior plane

\begin{tabular}{lcccccc}
\hline & \multicolumn{2}{c}{ Eyes without unintended initial dissection $(\mathrm{n}=234)$} & & Eyes with unintended initial dissection $(\mathrm{n}=29)$ & $\chi^{2}$ & \\
\cline { 2 - 3 } & Cases & $\%$ & & Cases & \% & \\
\hline Right eye & 126 & 53.85 & 7 & 24.14 & 9.1102 \\
Left eye & 108 & 46.15 & 22 & 75.86 & 0.003 \\
\hline
\end{tabular}

right eye $(\mathrm{P}=0.003)$ (Table 2). As shown in Table 3, significant differences were found in sphere $(\mathrm{T}=2.8, \mathrm{P}=0.006)$, SE ( $\mathrm{T}=2.37, \mathrm{P}=0.019), \mathrm{J} 0(\mathrm{~T}=2.05, \mathrm{P}=0.043)$ and axial length ( $\mathrm{T}=-2.79, \mathrm{P}=0.006)$ between eyes in which plane dissection was difficult and those in which it was not. Cylinder, J45, lenticular diameter, lenticular thickness, or corneal thickness were not significantly different between the two groups $(\mathrm{P}>0.05)$.

\section{Discussion}

Interface dissection difficulty is one of the most common intraoperative complications during SMILE procedures, especially when performed by relatively inexperienced surgeons (14). In this study, we tried to determine the factors associated with unintended dissection of the posterior plane so that caution can be exercised in such situations.

In the present study, the probability of unintended dissection of the posterior plane was $11.03 \%$ (29/263 eyes). In a statistical analysis performed by Hamed et al. (15) on 282 operated eyes, unintended dissection of the posterior plane resulting in lenticular adhesion to the corneal cap occurred in $2.12 \%$ of operated eyes. In another study by Shetty et al. (13), it occurred in $2 \%$ of 550 eyes operated on by an experienced surgeon. In yet another study (16), unintended posterior plane dissection occurred in 10/3,004 (0.33\%) eyes operated on by an experienced surgeon. In a statistical analysis of 1,800 eyes of 922 patients, Ivarsen et al. (17) reported that difficult lenticule extraction occurred in $1.9 \%$ of cases, and recommended timely termination upon the occurrence of suction loss and failed suction restoration attempts, in order to prevent subsequent difficulties in lenticule extraction. In this study, no obvious suction loss, black spot or opaque bubble layer affected the operation. As a surgeon who was newly acquainted with SMILE, the probability of unintended dissection of the posterior plane was similar to Titiyal et al. (12). Titiyal et al. (12) prospectively observed 100 cases of SMILE performed by a surgeon who was newly acquainted with the procedure, and difficulty in lenticule extraction was encountered in 9 cases (9\%), of which, 7 were caused by unintended posterior plane dissection.

To our knowledge, the difference in the incidence of unintended dissection of the posterior plane between left and right eyes has not been analyzed before. In the present 
Table 3 Comparisons of eyes with and without unintended initial dissection of the posterior plane

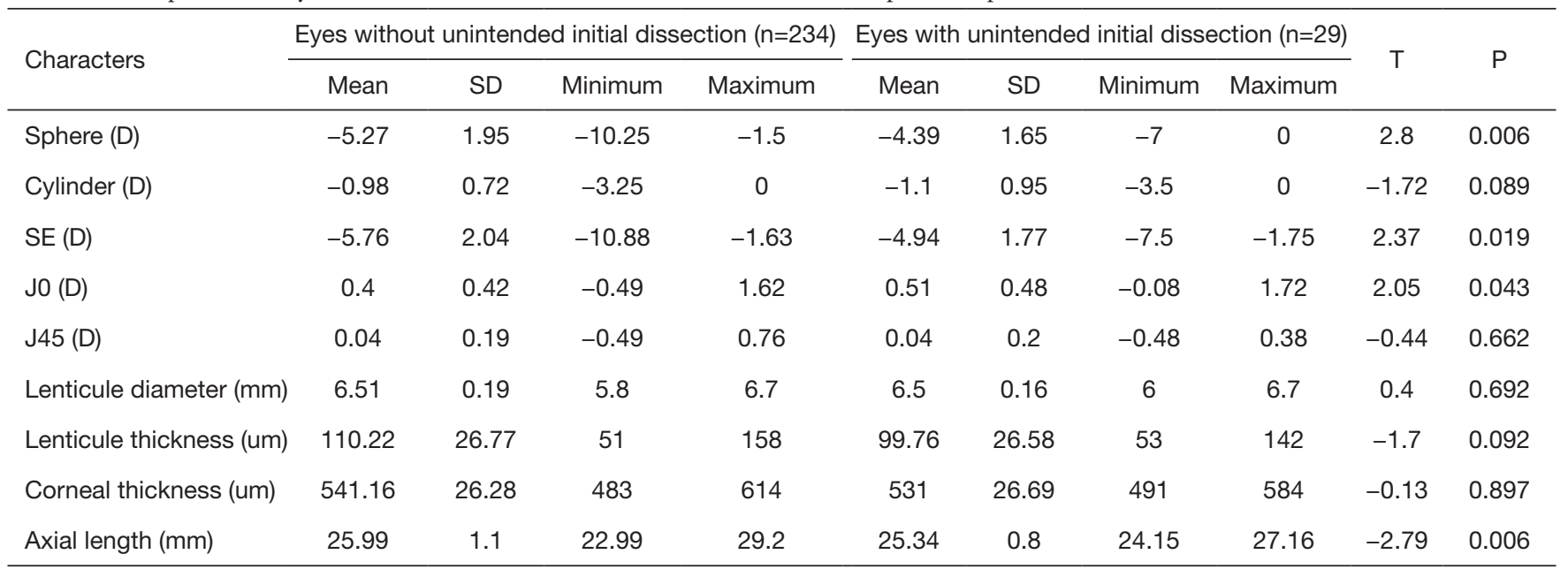

D, diopters; SE, spherical equivalent.

study, it was significantly more likely to occur in the left eye. Based on retrospective reviews of the surgical process, we speculate a possible reason that the patient's forehead and nose bridge disturb the position of the surgeon's right hand. The surgeon's right hand, which is holding the dissector, has to be elevated slightly more during dissection in left than in right eyes. Consequently, the right hand tends to move in a downward direction, which increases the chances of unintended posterior plane dissection. Therefore, it is recommended that surgeons who hold the dissector in their right hands should pay particular attention to hand position adjustments during dissection in the left eyes, to reduce the difficulty encountered during plane dissection. The result may potentially be different for a left-handed surgeon. Also, learning to use both hands can be helpful in preventing unintended dissection of the posterior plane.

In the aforementioned study of more than 1,500 eyes by Ivarsen et al. (17), no specific preoperative parameters were found for operated eyes in which plane dissection was difficult. However, in this study, difficulty in plane dissection during SMILE was encountered significantly more often in eyes with low SE, low sphere, high J0 or short axial length. Subsequent lenticular adhesion to the corneal cap were more likely to occur in Eyes with low SE, low sphere has also been observed by Shetty et al. (13) This may due to the lenticular thickness. In addition, in the current study, difficulty in plane dissection was more likely to be encountered in eyes with high $\mathrm{J} 0$ values (higher degrees of astigmatism at 90 degrees). This may be related to the astigmatic lenticular design model. High J0 may influence the peripheral lenticular thickness beside the small incision. Thin peripheral lenticular thicknesses increase the difficulty encountered during plane dissection (14).

Central Lenticular thickness was not a significant factor in the present study. The reason is that peripheral lenticular thickness besides the small incision is the key point rather than central lenticule thickness. The peripheral lenticule thickness of eyes with low SE or high J0 values is thin, thus plane dissection is challenging.

Other objective factors also cause difficulty in plane dissection, including abnormal laser energy, black spots, and the presence of an opaque bubble layer (17), while subjective factors include excessive eye movement. In addition, the dissection in this study was done through the superior incision directly without pocketing the two interfaces of the lenticular edge first. This might also lead to an increased incidence of unintended dissection of the posterior plane.

A limitation of the present study is that the surgery and practice experience of a single surgeon may differ from that of others. Moreover, for the benefit of patients, the surgeon started SMILE with a 2-mm incision. However, as unintended dissection of the posterior plane is a common complication, we are confident that all surgeons-especially novice surgeons-will benefit from the results of this study.

For novice surgeons, the selection of right eyes with high SE and low J0 values is recommended for the first few surgeries, and keep in mind that optical coherence tomography helps in identifying the separated lenticular planes (18-21). For experienced surgeons, extreme caution 
should still be exercised when performing SMILE on patients with low SE or high J0 values. In addition, learning to use both hands, and intra-operative optical coherence tomography (18), increasing minimum lenticule thickness for very thin lenticules (22) can be helpful in preventing unintended dissection of the posterior plane.

In conclusion, in the present study, difficulty in plane dissection during SMILE was encountered significantly more often in left eyes for a right-hand doctor, and in eyes with low SE or high J0 values. Special attention should be paid to prevent unintended initial dissection of the posterior plane in such situations.

\section{Acknowledgments}

The authors thanks Mr. Owen for polishing the manuscript. Funding: Supported in part by Shanghai Sailing Program (Grant No. 20YF1405000), the National Natural Science Foundation of China for Young Scholars (Grant No. 82000929 and 81700872), the National Natural Science Foundation of China (Grant No. 81770955), Joint research project of new frontier technology in municipal hospitals (Grant No. SHDC12018103), and the Project of Shanghai Science and Technology (Grant No. 17411950200).

\section{Footnote}

Reporting Checklist: The authors have completed the TRENT reporting checklist. Available at http://dx.doi. org/10.21037/atm-20-6759

Data Sharing Statement: Available at http://dx.doi. org/10.21037/atm-20-6759

Peer Review File: Available at http://dx.doi.org/10.21037/ atm-20-6759

Conflicts of Interest: All authors have completed the ICMJE uniform disclosure form (available at http://dx.doi. org/10.21037/atm-20-6759). The authors have no conflicts of interest to declare.

Ethical Statement: The authors are accountable for all aspects of the work in ensuring that questions related to the accuracy or integrity of any part of the work are appropriately investigated and resolved. The study was conducted in accordance with the Declaration of Helsinki (as revised in 2013). The study was approved by the institutional ethics board of Eye and ENT Hospital of Fudan University (No. ky2012-018) and written informed consent was taken from all the patients.

Open Access Statement: This is an Open Access article distributed in accordance with the Creative Commons Attribution-NonCommercial-NoDerivs 4.0 International License (CC BY-NC-ND 4.0), which permits the noncommercial replication and distribution of the article with the strict proviso that no changes or edits are made and the original work is properly cited (including links to both the formal publication through the relevant DOI and the license). See: https://creativecommons.org/licenses/by-nc-nd/4.0/.

\section{References}

1. Blum M, Lauer AS, Kunert KS, et al. 10-Year Results of Small Incision Lenticule Extraction. J Refract Surg 2019;35:618-23.

2. Han T, Xu Y, Han X, et al. Three-year outcomes of small incision lenticule extraction (SMILE) and femtosecond laser-assisted laser in situ keratomileusis (FS-LASIK) for myopia and myopic astigmatism. Br J Ophthalmol 2019;103:565-8.

3. Dong Z, Zhou X, Wu J, et al. Small incision lenticule extraction (SMILE) and femtosecond laser LASIK: comparison of corneal wound healing and inflammation. Br J Ophthalmol 2014;98:263-9.

4. Riau AK, Angunawela RI, Chaurasia SS, et al. Early corneal wound healing and inflammatory responses after refractive lenticule extraction (ReLEx). Invest Ophthalmol Vis Sci 2011;52:6213-21.

5. Mohamed-Noriega K, Riau AK, Lwin NC, et al. Early corneal nerve damage and recovery following small incision lenticule extraction (SMILE) and laser in situ keratomileusis (LASIK). Invest Ophthalmol Vis Sci 2014;55:1823-34.

6. Li M, Zhou Z, Shen Y, et al. Comparison of corneal sensation between small incision lenticule extraction (SMILE) and femtosecond laser-assisted LASIK for myopia. J Refract Surg 2014;30:94-100.

7. Li M, Zhao J, Shen Y, et al. Comparison of dry eye and corneal sensitivity between small incision lenticule extraction and femtosecond LASIK for myopia. PLoS One 2013;8:e77797.

8. Kobashi H, Kamiya K, Shimizu K. Dry Eye After Small Incision Lenticule Extraction and Femtosecond LaserAssisted LASIK: Meta-Analysis. Cornea 2017;36:85-91. 
9. Krueger RR, Meister CS. A review of small incision lenticule extraction complications. Curr Opin Ophthalmol 2018;29:292-8.

10. Tong JY, Cherepanoff S, Males JJ. SMILE Rescue: Delayed Lenticule Removal in a Patient With High Myopia. J Refract Surg 2017;33:199-202.

11. Sekundo W, Kunert KS, Blum M. Small incision corneal refractive surgery using the small incision lenticule extraction (SMILE) procedure for the correction of myopia and myopic astigmatism: results of a 6 month prospective study. Br J Ophthalmol 2011;95:335-9.

12. Titiyal JS, Kaur M, Rathi A, et al. Learning Curve of Small Incision Lenticule Extraction: Challenges and Complications. Cornea 2017;36:1377-82.

13. Shetty R, Negalur N, Shroff R, et al. Cap Lenticular Adhesion During Small Incision Lenticular Extraction Surgery: Causative Factors and Outcomes. Asia Pac J Ophthalmol (Phila) 2017;6:233-7.

14. Reinstein DZ, Carp GI, Archer TJ. The Surgeon's Guide to SMILE: Small Incision Lenticule Extraction. SLACK, Incorporated; 2018.

15. Hamed AM, Heikal MA, Soliman TT, et al. SMILE intraoperative complications: incidence and management. Int J Ophthalmol 2019;12:280-3.

Cite this article as: Zheng $\mathrm{K}$, Han T, Han Y, Liu F, Zhou X. Analysis of factors associated with unintended initial dissection of the posterior plane during small incision lenticule extraction. Ann Transl Med 2021;9(9):785. doi: 10.21037/atm-20-6759
16. Wang Y, Ma J, Zhang J, et al. Incidence and management of intraoperative complications during small-incision lenticule extraction in 3004 cases. J Cataract Refract Surg 2017;43:796-802.

17. Ivarsen A, Asp S, Hjortdal J. Safety and complications of more than 1500 small-incision lenticule extraction procedures. Ophthalmology 2014;121:822-8.

18. Zheng K, Han T, Zhao F, et al. Identification of separated lenticular planes using optical coherence tomography. Eur J Ophthalmol 2020;30:928-32.

19. Sharma N, Urkude J, Chaniyara M, et al. Microscopeintegrated intraoperative optical coherence tomographyguided small-incision lenticule extraction: New surgical technique. J Cataract Refract Surg 2017;43:1245-50.

20. Zheng K, Xu Y, Han T, et al. Five Signs of Unintended Initial Dissection of the Posterior Plane During SMILE. J Refract Surg 2018;34:69-70.

21. Titiyal JS, Rathi A, Kaur M, et al. AS-OCT as a Rescue Tool During Difficult Lenticule Extraction in SMILE. J Refract Surg 2017;33:352-4.

22. Siedlecki J, Luft N, Keidel L, et al. Variation of Lenticule Thickness for SMILE in Low Myopia. J Refract Surg 2018;34:453-9. 